\section{Hollow polymer particles that are pH-responsive and redox sensitive: two simple steps to triggered particle swelling, gelation and disassembly $\dagger$}

\author{
Robert Bird, ${ }^{a}$ Tony J. Freemont ${ }^{b}$ and Brian R. Saunders ${ }^{* a}$ \\ Received 28th October 2010, Accepted 29th November 2010 \\ DOI: $10.1039 / \mathrm{c0cc04657d}$ \\ A new, simple, two-step method is introduced for preparing \\ hollow particles that are both $\mathrm{pH}$-responsive and redox sensitive. \\ Hollow poly(methyl methacrylate-co-methacrylic acid) particles \\ swell at moderate $\mathrm{pH}$ values, form gels in concentrated dispersions \\ and can be disassembled by adding reducing agents.
}

Hollow polymer particles continue to attract major interest because they have potential applications in drug delivery, catalysis, electronic materials, lightweight fillers, artificial cells, vessels for confined reactions, gene delivery, encapsulation of cosmetics and paints. ${ }^{1} \mathrm{We}$ are interested in these dispersions from the viewpoint of combining reversible fluid-to-gel transitions with delivery. It is the presence of an empty (fillable) core that offers advanced delivery and encapsulation possibilities. For delivery applications a drug, gene, etc. must be protected from body fluids until release is triggered. ${ }^{2}$ Seminal studies in the area of hollow polymer particles were conducted by Caruso et al. ${ }^{3}$ An emerging area is stimulus responsive hollow polymer particles which give externally triggered dimension changes in response to $\mathrm{pH}$ or temperature change. ${ }^{4,5}$ These responsive hollow particles provide new opportunities for on-demand delivery applications. The reports involving hollow polymer particles have almost exclusively relied on colloid templating. ${ }^{1,3-12}$ That method typically requires assembly of a polymer onto a colloid particle template followed by dissolution of the template to give hollow particles. When silica is used as the template, which is often the case, HF etching is required for its removal. Although colloid templating is good at providing low polydispersity size distributions it is a multistep process involving hazardous reagents (e.g., HF) and is not ideally suited for large scale preparation. Other approaches involve in situ polymerisation, e.g., polymerisation within vesicles. $^{13}$ Related to this coacervation approaches have also been used to generate complex geometries. ${ }^{14}$ A simple, scalable, approach for preparing hollow responsive polymer particles without the need for colloid templates is currently lacking.

${ }^{a}$ School of Materials, Manchester University, Grosvernor St, Manchester, M139PL,UK.E-mail: b.saunders@manchester.ac.uk; Tel: + 44 (0) 1613065944

${ }^{b}$ School of Medicine, Manchester University, Oxford Rd, M13 9PT, $U K$

$\dagger$ Electronic supplementary information (ESI) available: Experimental details and additional images. See DOI: 10.1039/c0cc04657d

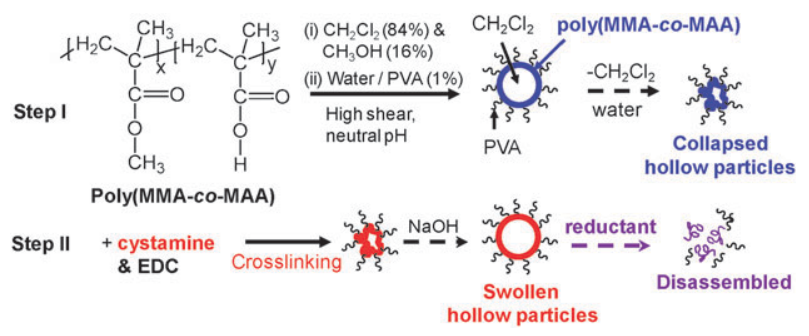

Scheme 1 Schematic representation of the two-step method used to prepare reversibly crosslinked hollow $\mathrm{pH}$-responsive particles.

Scheme 1 illustrates the new approach used here to prepare reversibly crosslinked $\mathrm{pH}$-responsive hollow poly(MMA-coMAA) (methyl methacrylate and methacrylic acid) particles. Our method (ESI $\dagger$ ) involves preparing hollow particles and then crosslinking them via cystamine grafted onto the shells using EDC. In Step I the copolymer was dissolved in mixed $\mathrm{CH}_{2} \mathrm{Cl}_{2} / \mathrm{CH}_{3} \mathrm{OH}$ solvent and the solution added to a polyvinylalcohol (PVA)/water solution under high shear. $\mathrm{CH}_{3} \mathrm{OH}$ rapidly diffused out of the $\mathrm{CH}_{2} \mathrm{Cl}_{2}$ droplets into the aqueous phase leaving the $\mathrm{CH}_{2} \mathrm{Cl}_{2}$ behind and the insoluble polymer to precipitate at the droplet/water interface. The latter formed a shell around the $\mathrm{CH}_{2} \mathrm{Cl}_{2}$ droplets. As the $\mathrm{CH}_{2} \mathrm{Cl}_{2}$ slowly evaporated the shell collapsed. The shell was then reversibly crosslinked with cystamine (Step II) to give reversibly crosslinked (flexible) $\mathrm{pH}$-swellable particles. We propose that it is the amphiphilic nature of the copolymer that enables preparation of hollow polymer particles in this way.

Poly(MMA-co-t-BMA) (tert-butyl methacrylate) was prepared using solution free-radical polymerisation (ESI $\dagger$ ). The copolymer had a $M_{\mathrm{n}}$ of $27300 \mathrm{~g} \mathrm{~mol}^{-1}$, polydispersity of 1.9 and contained $40 \mathrm{~mol} \%$ of $t$-BMA as deduced from ${ }^{1} \mathrm{H}$ NMR (Fig. S1, ESI $\dagger$ ). Acid hydrolysis of poly(MMA-co$t$-BMA) in dioxane gave poly(MMA-co-MAA). The ${ }^{1} \mathrm{H}$ NMR spectrum of the latter showed good conversion of $t$-BMA groups to MAA (Fig. S2, ESI $\dagger$ ). Potentiometric titration data (Fig. S3, ESI $\dagger$ ) showed that the copolymer contained $39 \mathrm{~mol} \%$ MAA.

We first consider non-crosslinked collapsed hollow poly(MMA-co-MAA) particles (Step I). Optical micrographs were taken shortly after high-shear mixing (emulsification) and also after $\mathrm{CH}_{2} \mathrm{Cl}_{2}$ evaporation. Fig. 1(a) shows that a shell of polymer formed around the $\mathrm{CH}_{2} \mathrm{Cl}_{2}$ droplets just after emulsification. Partial evaporation of the $\mathrm{CH}_{2} \mathrm{Cl}_{2}$ caused the 


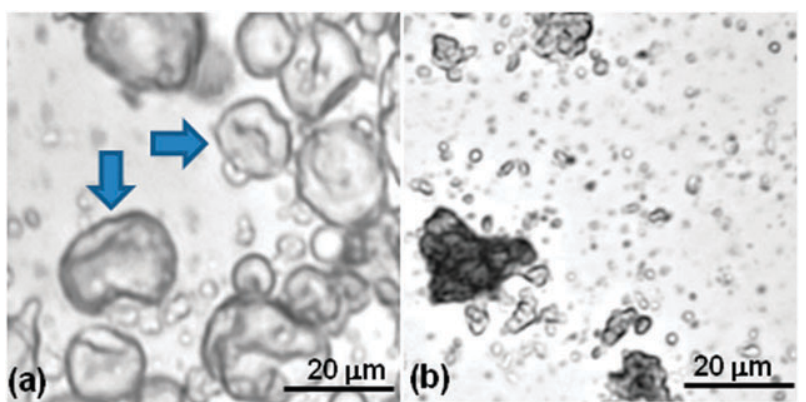

Fig. 1 Optical micrographs of (a) poly(MMA-co-MAA)-coated $\mathrm{CH}_{2} \mathrm{Cl}_{2}$ droplets just after preparation and (b) particles after $\mathrm{CH}_{2} \mathrm{Cl}_{2}$ evaporation.

shells to begin to collapse. After the $\mathrm{CH}_{2} \mathrm{Cl}_{2}$ had evaporated the shells completed their collapse (Fig. 1(b)), which is reversible (later). Because of the turbulent shear flow present during high-shear mixing there was a range of sizes for the collapsed particles. The largest collapsed particles were in the region of $20 \mu \mathrm{m}$; whereas, the smallest were less than $1 \mu \mathrm{m}$.

We tested whether the amphiphilic nature of the copolymer was important for shell formation and prepared particles using poly(MMA-co- $t$-BMA). That copolymer was fully soluble in $\mathrm{CH}_{2} \mathrm{Cl}_{2}$ and $\mathrm{CH}_{3} \mathrm{OH}$ (a non-solvent for that copolymer) was not added. The poly(MMA-co- $t$-BMA) particles were smaller, average size of $1.5 \mu \mathrm{m}$, spherical and uniform after $\mathrm{CH}_{2} \mathrm{Cl}_{2}$ evaporation (Fig. S4, ESI $\dagger$ ). Poly(MMA-co- $t$-BMA) is hydrophobic and, as a consequence, was confined to the $\mathrm{CH}_{2} \mathrm{Cl}_{2}$ droplet interior. This prevented shell formation. The experiment indicates the ability to form hollow poly(MMA-co-MAA) is a consequence of the polar MAA segments.

Potentiometric titration data for the non-crosslinked hollow poly(MMA-co-MAA) particles (Fig. S3(a), ESI $\dagger$ ) showed that the $\mathrm{pH}$ increased at first and then decreased upon addition of $\mathrm{NaOH}$, before finally increasing again near $100 \%$ neutralisation. By comparison the $\mathrm{pH}$ change for MAA-containing microgels usually shows increasing and plateau regions. ${ }^{15}$ Optical microscopy showed that this behaviour (maximum in $\mathrm{pH}$ followed by a minimum) for our particles coincided with expansion of the collapsed particles. The decrease in $\mathrm{pH}$ upon adding further $\mathrm{NaOH}$ (after the maximum) is due to a major structural transition that exposed inner parts of collapsed polymer particles and presented additional, non-neutralised, MAA segments. This process exposed buried MAA segments and caused a self-buffering response for dispersions containing high particle concentrations. The average $\mathrm{p} K_{\mathrm{a}}$ for the poly(MMA-co-MAA) particles was 7.3 which is a relatively high $\mathrm{p} K_{\mathrm{a}}$ compared to that of poly(MAA) (ca. 6). This is a consequence of copolymerisation of MAA with MMA. The dispersions became transparent (complete particle dissolution) when the $\mathrm{pH}$ was greater than the $\mathrm{p} K_{\mathrm{a}}$ and the degree of neutralisation exceeded 50\% (Fig. S3(b), ESI $\dagger$ ). The combination of the ability to protect buried copolymer segments, complete dissolution at high neutralisation and construction from established biomaterial components may lead to delivery applications for these hollow, non-crosslinked particles.

We next introduced reversible crosslinking into the hollow collapsed particles (Step II). Elemental analysis showed that the cystamine functionalised poly(MMA-co-MAA/Cyst) particles contained $3.8 \mathrm{~mol} \%$ cystamine (ESI $\dagger$ ). This corresponds to functionalisation of $c a .10 \%$ of the $\mathrm{RCOOH}$ groups. SEM images of the collapsed poly(MMA-co-MAA/ Cyst) particles deposited at a $\mathrm{pH}$ of 6 are shown in Fig. 2(a). The flexible shells folded inwards on themselves. The folded/collapsed particle morphology is accessible over a wide size range, i.e., from $c a$. $500 \mathrm{~nm}$ to $5 \mu \mathrm{m}$. If required the particles could be fractionated using centrifugation.

Optical microscopy was used to track the pH-triggered swelling of collapsed particles as a high $\mathrm{pH}$ water front infiltrated isolated particles initially dispersed in neutral water (in the collapsed state), see Fig. 2(b). The structural transition starts from one side of the particle (in contact with the high $\mathrm{pH}$ water front) and spread rapidly throughout the particle. These images clearly demonstrate $\mathrm{pH}$-triggered swelling of collapsed particles and the excellent flexibility of the shells. Optical microscopy images for a number of swollen particles show that they are hollow (Fig. S5, ESI $\dagger$ ).

Particles were also prepared using pyrene methylamine $\mathrm{HCl}$ as a chromophore to probe the crosslinker distribution. The chromophore was incorporated during cystamine functionalisation using the same EDC coupling reaction. It is logical to assume similar distributions of chromophore and cystamine within the particles. A representative fluorescence microscopy image (Fig. 2(c)) shows the chromophore to be confined to the shells. These data confirm that the swollen particles are hollow and cystamine penetrated the shells.

In order to measure the $\mathrm{pH}$-dependence of particle swelling, individual particles were tracked using optical microscopy as buffer $(0.1 \mathrm{M})$ solutions flowed across the viewing plane. Because these dispersions had low particle concentrations the $\mathrm{pH}$ values were constrained to those of the added buffer.
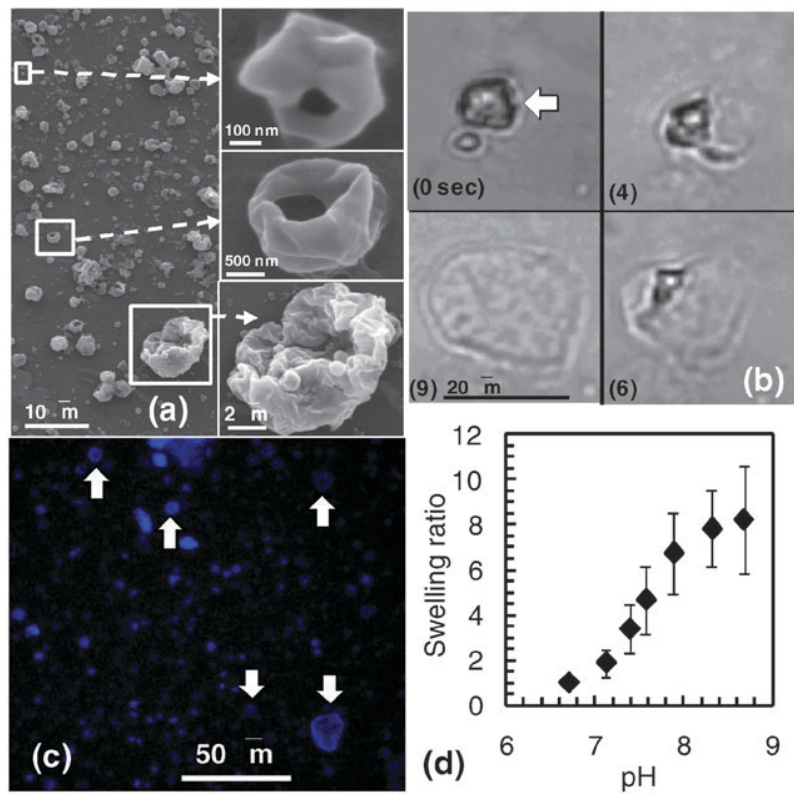

Fig. 2 (a) SEM images of reversibly crosslinked poly(MMA-coMAA/Cyst) particles. (b) Swelling of a particle after exposure to high $\mathrm{pH}$ water (time shown in seconds). (c) Fluorescence microscopy images for pyrene labelled particles $(\mathrm{pH}=9.5)$. (d) Swelling ratio as a function of $\mathrm{pH}$. 


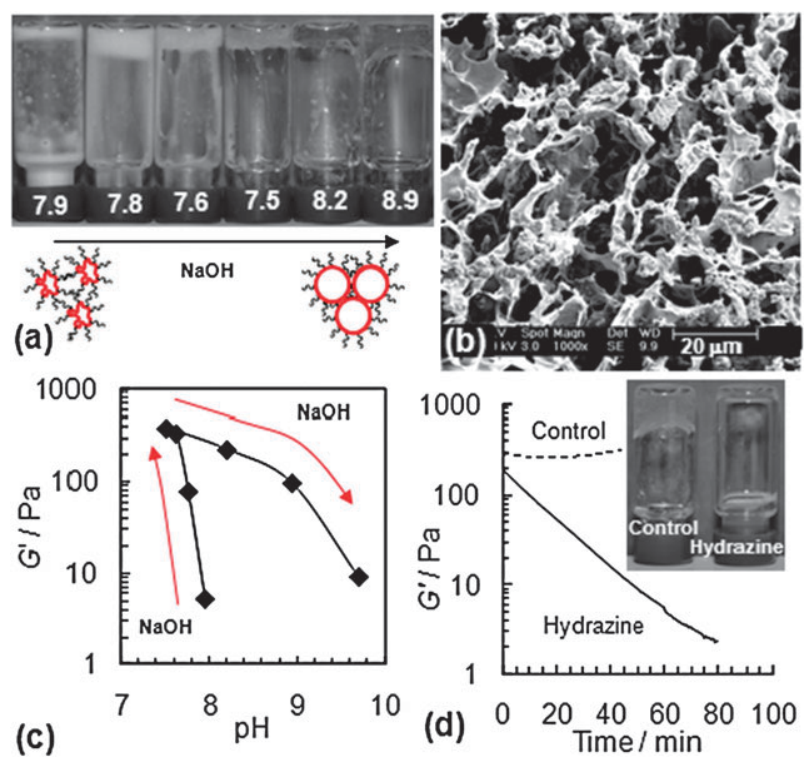

Fig. 3 (a) Fluid-to-gel transition at different $\mathrm{pH}$ values (shown). (b) SEM images of freeze-dried gel. The $\mathrm{pH}$ was $c a$. 8.5. (c) Variation of $G^{\prime}$ with $\mathrm{pH}$ (5 wt $\%$ dispersion). (d) $G^{\prime}$ with time for a $5 \mathrm{wt} \%$ dispersion at $\mathrm{pH}=7.5$ with added hydrazine $(0.05 \mathrm{M})$. The inset shows images of the tubes.

It can be seen (Fig. 2(d)) that swelling occurs when the $\mathrm{pH}$ reaches 7.1 and increases with $\mathrm{pH}$. Swelling ratios of up to about 8 were measured. Therefore, substantial swelling is expected at physiological $\mathrm{pH}$ (7.4) for these hollow particles.

The ability of glutathione (GT), an important naturally occurring reductant, to trigger disassembly of the swollen state of the hollow particles was tested. GT exposure caused disassembly of the aggregates in the swollen state $(\mathrm{pH}=9.5)$ and the remnants of shells can be seen from the SEM image (Fig. S6, ESI $\dagger$ ). These data demonstrate GT-triggered disassembly of these $\mathrm{pH}$-responsive hollow particles.

We next investigated the ability of concentrated poly(MMA-co-MAA/Cyst) dispersions to show $\mathrm{pH}$-triggered gel formation (see Fig. 3(a)). Particulate gels first formed (at $5 \mathrm{wt} \%$ ) when the $\mathrm{pH}$ exceeded 7.9 and were then stable at $\mathrm{pH}=7.5$ (For these concentrated dispersions the $\mathrm{pH}$ decreased on adding sufficient $\mathrm{NaOH}$ to trigger particle swelling). The gel turbidity decreased with increasing $\mathrm{NaOH}$ concentration due to further shell swelling. SEM investigation of the gels (Fig. 3(b)) showed an expanded structure with deformed spherical pores. These are remnants of hollow swollen particles that were in contact and is the space-filling structure responsible for gel formation. A higher magnification images is shown in Fig. S7 (ESI $\dagger$ ). These micrometre-scale pores are a good size to promote cell growth and the morphology is reminiscent of polymerised high internal phase emulsions. ${ }^{16}$ However, our materials consist of $\mathrm{pH}$-responsive polymer particles and represent a new class of hydrogel, i.e., hollow particle gels.

Dynamic rheology was used to probe the mechanical properties of the gels. (see Fig. 3(c)). The storage modulus shows a maximum $G^{\prime}$ close to $\mathrm{pH}=7.5$. A minimum $\tan \delta$ was also evident in this $\mathrm{pH}$ region (see Fig. S8, ESI $\dagger$ ). There is large hysteresis with respect to $\mathrm{pH}$ which implies a permanent $\mathrm{pH}$-triggered structural change. These data are consistent with the base-triggered exposure of buried MAA groups noted above. The addition of $\mathrm{NaOH}$ causes unfolding (and swelling) of the collapsed hollow particles (Fig. 2(b)) which sterically repel neighbouring particles through excluded volume effects producing a finite network elasticity. At $\mathrm{pH}$ values greater than 7.5 the ionic strength will increase strongly for these concentrated dispersions decreasing electrostatic repulsion between $\mathrm{MAA}^{-}$groups. The continued decrease in $G^{\prime}$ at $\mathrm{pH}$ approaching 10 may indicate shell fragmentation. It is stressed that the particulate gels showed good stability at $\mathrm{pH}=7.5$ for extended times (weeks). This is a convenient $\mathrm{pH}$ region for physiological applications.

We also tested redox-triggered gel-breakdown using hydrazine. This reductant was chosen to demonstrate the generality of triggered disassembly. Added hydrazine caused an immediate $G^{\prime}$ decrease which continued with time (Fig. 3(d). The value for $\tan \delta$ increased with time (Fig. S9, ESI $\dagger)$. This is the first example of redox triggered breakdown of a pH-responsive particle gel to our knowledge. Tube inversion (inset of Fig. 3(d)) demonstrates visually that addition of hydrazine caused a gel-to-fluid transition.

Our new, simple, two-step method for preparing hollow $\mathrm{pH}$-responsive particles has enabled preparation of space filling gels with microporous (inter-connected) porosity. The particles and gels are degradable using reductants. An advantage is that the particles were prepared without the use of small molecule surfactants. A further advantage of this method is that multi-gram scale quantities of hollow particles can rapidly be prepared and it is amenable to scale up. This new approach should be well suited for loading of the hollow particles with a large range of actives that are soluble, or dispersible, in $\mathrm{CH}_{2} \mathrm{Cl}_{2}$ and providing $\mathrm{pH}$ - or redox-triggered release. It is potentially useful for regenerative medicine applications where in situ gel formation is required. ${ }^{17} \mathrm{We}$ expect this approach to be generally applicable to a wide of amphiphilic copolymers containing carboxylic acid groups.

\section{Notes and references}

1 G. Sukhorukov, A. Fery and H. Mohwald, Prog. Polym. Sci., 2005, 30, 885.

2 C. Tsitsilianis, Soft Matter, 2010, 6, 2372.

3 F. Caruso, R. A. Caruso and H. Mohwald, Science, 1998, 282, 1111.

4 G. Li, G. Liu, E. T. Kang, K. G. Neoh and X. Yang, Langmuir, 2008, 24, 9050.

5 A. Zelikin, Q. Li and F. Caruso, Chem. Mater., 2008, 20, 2655.

6 D. Chen and M. Jiang, Acc. Chem. Res., 2005, 38, 494.

7 G. Li, Q. Shi, K. G. Neoh, E. T. Kang and X. Yang, Chem. Mater., 2010, 22, 1309.

8 M.-K. Park, K. Onishi, J. Locklin, F. Caruso and R. C. Advincula, Langmuir, 2003, 19, 8550.

9 D. G. Shchukin, G. Sukhorukov and H. Mohwald, Angew. Chem., Int. Ed., 2003, 42, 4471.

10 M. Yang, J. Ma, C. Zhang, Z. Yang and Y. Lu, Angew. Chem., Int. Ed., 2005, 44, 6727.

11 L. Zha, Y. Zhang, W. Yang and S. Fu, Adv. Mater., 2002, 14, 1090.

12 Y. Zhang, M. Jiang, J. Zhao, Z. Wang, H. Dou and D. Chen, Langmuir, 2005, 21, 1531.

13 M. Sauer and W. Meier, Chem. Commun., 2001, 55.

14 H. N. Yow and A. F. Routh, Soft Matter, 2006, 2, 940.

15 B. E. Rodriguez, M. S. Wolfe and M. Fryd, Macromolecules, 1994, 27, 6642 .

16 A. Barbetta and N. R. Cameron, Macromolecules, 2004, 37, 3202.

17 J. M. Saunders, T. Tong, C. L. Le Maitre, T. J. Freemont and B. R. Saunders, Soft Matter, 2007, 3, 486-494. 